\title{
Is functional outcome different in posterior and anterior circulation stroke?
} \author{
${ }^{6}$ Michael Knoflach, ${ }^{3}$ Stefan Greisenegger. \\ 1Department of Neurology, Krankenanstalt Rudolfstiftung Vienna, Austria; \\ 2Danube University Krems \& Gesundheit Österreich GmbH/BIQG, Vienna, Austria; \\ ${ }^{3}$ Department of Neurology, Medical University of Vienna, Austria; \\ ${ }^{4}$ Department of Neurology, Krankenhaus Barmherzige Brüder, Vienna, Austria \\ ${ }^{5}$ Department of Neurology 2, Kepler Universitätsklinikum, Med Campus III, Linz, Austria \\ ${ }^{6}$ Department of Neurology, Medical University of Innsbruck, Austria;
}

${ }^{1}$ Peter Sommer, ${ }^{2}$ Alexandra Posekany, ${ }^{3}$ Wolfgang Serles, ${ }^{3}$ Martha Marko, ${ }^{3}$ Sebastian Scharer ${ }^{1}$ Elisabeth Fertl, ${ }^{4}$ Julia Ferrari, ${ }^{4}$ Wilfried Lang, ${ }^{5}$ Milan Vosko, ${ }^{3}$ Simon Szabo, ${ }^{6}$ Stefan Kiechl,

Background: Posterior circulation stroke (PCS) account for $20 \%$ of all ischemic strokes. There is limited evidence whether functional outcome of PCS is comparable to that of anterior circulation stroke (ACS). Recently, we reported prolonged timeto-thrombolysis in patients with PCS in a large nationwide cohort of patients with acute ischemic stroke. The aims of our present study were first to analyze whether functional outcome differs between patients with PCS and anterior circulation strokes (ACS), and second whether prolonged prehospital delays and infarct localization influence the functional outcome.

Methods: Patients with acute ischemic stroke prospectively enrolled within the Austrian Stroke Unit Registry (ASUR) were stratified by infarct localization according to the Oxfordshire Community Stroke Project Classification. Propensity score matching was used to control for covariate imbalances and to match patients with PCS and ACS. Patients were matched for stroke severity, rtPA-treatment, demographic and vascular risk factors. Main Outcomes were the distribution of mRS after 3 months and multiple proportional odds models to estimate the influence of the infarct localization on the functional outcome.

Results: From a total of 90484 patients enrolled within the ASUR, 9208 (4604 PCS/4604 ACS) were matched, of those 954 (477 in each group) were treated with IVT. We detected a significant shift towards better 3-month functional outcome in patients with ACS compared to PCS (OR 1.19, 95\%CI 1.1-1.28, $\mathrm{p}<0.0001$ ). In particular, functional outcome was worse in PCS with onset-to-door-time (ODT)>270 minutes (OR 1.34, 95\% $\mathrm{Cl}$ 1.17-1.54, $\mathrm{p}<0.0001$ ) or in PCS with unknown ODT (OR 1.26, $95 \% \mathrm{Cl} 1.13-1.42, \mathrm{p}<0.0001)$, however, we did not detect any difference in functional outcome between ACS and PCS in patients with an ODT $\leq 270$ minutes (1-180 minutes: OR 0.92, 95\% Cl 0.78-1.09, $\mathrm{p}=0.3554 ; 181-270$ minutes: OR 1.04, 95\%Cl: 0.79-1.37, $\mathrm{p}=0.7689$; figure). In patients treated with IVT, functional outcome was not significantly different between PCS and ACS.
Conclusions: PCS was associated with worse outcome compared to ACS in patients arriving later than 4.5 hours at hospital or in those with unknown onset of symptoms. Our results urge for implementation of preclinical patient-triage tools involving symptoms of the posterior circulation.

Figure: multivariate proportional odds regression model demonstrating odds ratios (OR) for worse functional outcome after three months stratified by the onset-to-door-time (ODT) in minutes.

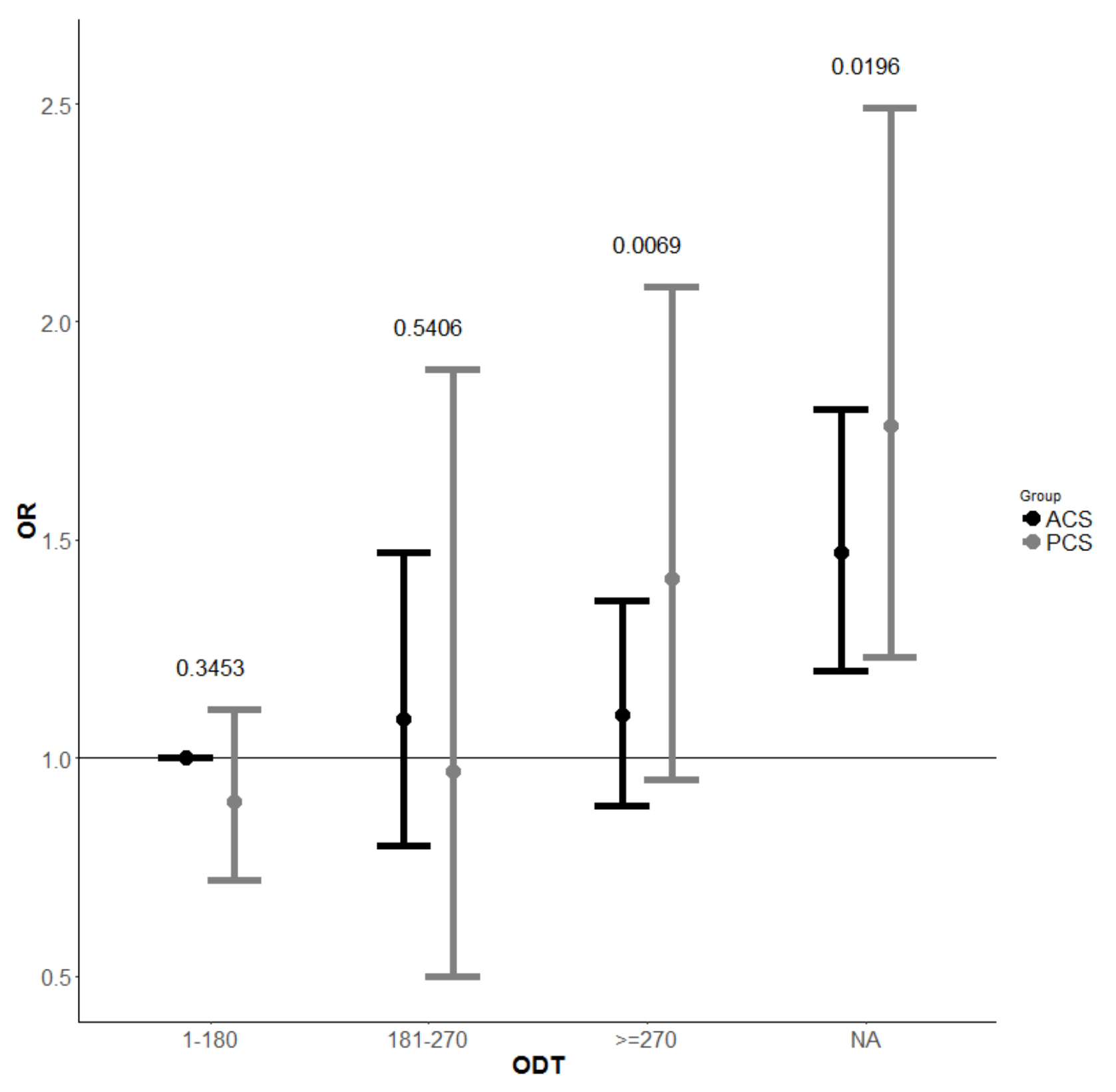

Boxes represent odds ratios and $95 \%$ confidence intervals. The model was adjusted for the following variables: infarct localization, NIHSS, age, sex, ODT, pre-stroke mRS, rtPA-treatment, hypertension, hyperlipidemia, diabetes and etiology according to the TOAST-classification. Reference levels for this model were: stroke localization: anterior circulation stroke (ACS), ODT: 1-180 minutes. 